\title{
Modular soft mechatronic manipulator for minimally invasive surgery (MIS): overall architecture and development of a fully integrated soft module
}

\author{
Giada Gerboni $(\mathbb{D} \cdot$ Tommaso Ranzani $\cdot$ Alessandro Diodato • \\ Gastone Ciuti - Matteo Cianchetti - Arianna Menciassi
}

Received: 1 November 2014/ Accepted: 8 August 2015/Published online: 7 September 2015

(C) The Author(s) 2015. This article is published with open access at Springerlink.com

\begin{abstract}
This paper describes the design and development of a modular soft manipulator for minimally invasive surgery, which equals the high dexterity of classic hyper redundant continuum, but rigid, robots resulting in safer potential interaction with internal organs. The manipulator relies on the use of a soft flexible fluidic actuator in each of its modules, which can be wireless controlled by means of an embedded fluidic control unit. This actuation unit is equipped with three miniaturized latching valves, a wireless microcontroller board, and a specifically designed fluidic distributor integrated into the elastomeric material that the module is made of. FEM simulations and experimental tests verified the reliability of the distributor in acting as a piping system inside each module. The mobility of the fully integrated soft module was evaluated in terms of static performances and covered workspace. The module's dynamic model during one-chamber motion was estimated from the parameter estimation analysis. The characterization of the single module behaviour is intended as first step to ease the future high level control of the multi-modular architecture.
\end{abstract}

G. Gerboni $(\bowtie) \cdot$ T. Ranzani · A. Diodato

G. Ciuti - M. Cianchetti - A. Menciassi

The BioRobotics Institute - Scuola Superiore Sant'Anna, Viale Rinaldo Piaggio 34, 56025 Pontedera, Pisa, Italy

e-mail: g.gerboni@sssup.it
Keywords Flexible fluidic actuators - Soft robotics · Modular robot $\cdot$ Minimally invasive surgery $\cdot$ Wireless control

\section{Introduction}

The minimally invasive surgery (MIS) theatre is one of the most challenging environments for the maneuverability of operating tools, which are required to reach remote anatomical areas via very intricate paths [1]. Due to the presence of other organs on the surgical path (Fig. 1), a laparoscopic manipulator, which enters directly from a localized skin incision (or from a natural orifice in the case of, natural orifice translumenal endoscopic surgery (NOTES) [2]) has to assume different curvatures in order to overcome obstacles and approach the surgical target with an optimal orientation of the end-effector with respect to the surgical target. Given the variety of surgical tasks and procedures, MIS instruments need to be very flexible and easily adaptable to varied situations [3].

Conventional rigid joint-link manipulators have limitations in terms of dexterity and kinematic configurations, which make some surgical procedures impossible to be performed entirely with the same instrument. Continuum-like robots, instead, offer the possibility of building manipulators with a hyper redundant number of degrees of freedom (DOFs), which are able to reach a certain target with multiple shapes and without kinematic singularities [4]. These 




Fig. 1 Left Envisaged modular and soft system for MIS. Right Flexible fluidic actuator (FFA) general working principle

structures act like snakes or trunks, which are the source of inspiration in most continuum robots [5, 6]. However, hyper redundant robots are characterized by important issues regarding the complexity associated with the increasing number of DOFs.

Modular robotics has traditionally been proposed as an alternative approach to deal with the fabrication, assembly and actuation of hyper redundant robots [7, 8]. The basic principle of modular robotics involves a set of elementary blocks that connect/disconnect together to create highly articulated structures. The strength of modular robotics relies on the possibility of obtaining the best outcomes from any kind of assembly of its basic units (i.e the modules). Therefore, the capabilities of the final structure can be quite arbitrarily changed without any re-design. This aspect has attracted increasing interest in the medical field (particularly in MIS) where modularity and related flexibility allow the surgical device to overcome, or at least to circumvent, most of the anatomical impediments in surgical procedures [9].

Depending on the number of modules, the workspace of the manipulator can be enlarged, thus additional robot configurations, reaching the same target with the end effector, become possible. Examples of modular endoluminal robots as in $[10,11]$ show how modularity can help in both the construction and actuation of surgical robotic platforms entirely built inside the abdominal cavity by small wireless controllable units that are docked together, after being delivered through a natural orifice access (i.e. the oral cavity). Given the highly constrained environment, MIS manipulators are very likely to come into close contact with anatomical organs and tissues, while moving towards the surgical operation site. Therefore, safe interaction between the instrument and the internal organs is of great importance. This is one of the main reasons why research in robotics is recently moving towards soft mechatronic technologies [1214].

The use of compliant and highly deformable materials enables us to completely rethink the entire bodyware of the system, which unlike rigid link machines, has a synergic distribution of sensing and actuating components integrated all over the actuator's body. In addition, thanks to the compliance of the materials used, soft devices could be intrinsically safe and adaptable in the case of un-structured working environments.

Traditionally, in robotics adaptability is taken into account just at the control level, by complex software for modeling and planning, which enable rigid link robots to become safer when in contact with external objects $[15,16]$. In soft robotics, the flexibility can be already embodied in the robotic structures, thus a soft and safe interaction is guaranteed by the hardware itself [17]. Soft-bodied robots are intrinsically safer than rigid link robots, as, in the case of contact (or even crashes) with external objects, they are more likely to deform and, possibly, even adsorb much of the energy involved in the contact itself. This thus decreases the risk of damage when approaching delicate structures, such as organs, nerves, and vessels [18].

Among the various and emerging actuation methods for soft robots, fluidic actuation of flexible structures has a high potential in medical applications. Flexible fluidic actuators (FFAs) are compliant systems with deformation driven by fluid pressure [19]. The use of inert fluids (air or water) makes them intrinsically safer for medical applications than those actuators that require highly energized parts. Figure 1, shows how a cylindrical elastomeric body can either be bent or elongated depending on the level of fluid pressure in each chamber. FFAs have far fewer parts than traditional robotic actuators, such as motors [20, 21], SMA (Shape Memory Alloys) based actuators [22] and piezo-electric actuators [23], thus their susceptibility to wear and tear is considerably lower.

However, fluidically driven soft actuators, such as the FFAs, present lots of difficulties in terms of modularity, since currently available fluidic power sources and regulators are not yet configured to be properly integrated into a soft module. To circumvent this limitation this work proposes a re-design of a conventional fluidic system to allow both the 
employment of standard fluidic components and the effective realization of a modular soft manipulator already sized for MIS applications. In the next section the paper presents a detailed discussion of the design strategies proposed to tackle with the modularity of soft robots and proposes a system architecture design, whose modules incorporate a 3DOFs FFA and which completely fulfills all the requirements of modular robotics: in fact it consists of a fluidic system that does not depend on the number of employed modules. The adopted strategy to control each module, without affecting the modularity of the system, is the use of a single fluidic supply line for the whole manipulator combined with wireless control of small valves integrated into each module. The design and fabrication of the single module are described in detail in Sect. 3. The paper concludes with the static and dynamic performances of the single module (Sects. 4 , 5) demonstrating the feasibility and the potentialities of the integrated wireless control of a fully modular soft manipulator.

\section{System architecture}

The general architecture of a serial modular robot (Fig. 2) consists of a set of $N_{M}$ modules each containing $N_{A}$ actuators, which are under the control of a single central system. All the actuators need to be reached by the power and by the control signal. The power line (blue line) and the control line (red line) are delivered by the central system, which normally controls the set of modules (Fig. 2) [11]. In the



Fig. 2 General architecture of a modular robotic system specific case of actuators that exploit fluidic power (be it pneumatic or hydraulic), the actuation is regulated by valves, which control the pressure/flow of a fluid towards the FFAs. The fundamental principle of modular robotics dictates that both the central system and each module must be minimally affected by the number of modules in the whole system. Considering an architecture with modules positioned in a snakelike configuration, as in the current case, an extremely limiting factor is the total number of lines, which need to run from the central system towards the modules passing through the robot structure. If all the lines are passed inside the modules, there would be a significant difference between the most proximal one to the central system module and the most distal, since the first module would allocate the space for all the lines of the entire set (Fig. 2).

To explain how the system is affected by the number of modules in different control approaches in modular robots, Fig. 3 presents the dependence of the number of lines $N_{L}$, on the number of modules $N_{M}$. A basic control of a modular architecture, shows the significant dependence on the number of modules, as each module is reached by separate lines of both fluidic power and control (as in Fig. 2). If we consider the lines passing through the modules, this method is heavily affected by $N_{M}$ (Fig. 3). In fluidic actuated systems, one of the most common approaches is the centralized control, where the fluidic power is delivered to each module's actuator separately and controlled by the central system. Examples can be found in serial soft manipulators as in [18] and soft gaiting robots as in [24]. In these robots the valves are placed

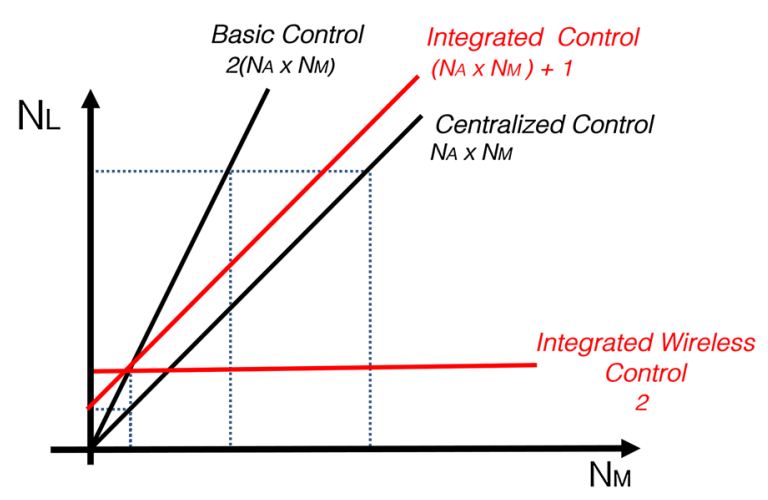

Fig. 3 Dependence of the number of lines connecting the modules to the central systems $\left(N_{L}\right)$ to respect to the number of modules $\left(N_{M}\right)$ 
remotely from the group of fluidic actuated modules and are directly addressed by the central system control. With the valves positioned away from the modules, the amount of independently controlled power lines for each actuator counts for the total number of lines NL in the system (Fig. 3). The dependence of $N_{L}$ on $N_{M}$ is again linear with a unitary coefficient, which still presents several limitations in terms of the maximum number of modules that can be practically employed. The integrated control strategy consists of the incorporation of all valves related to the module's actuators inside the module itself. This means that just one single fluidic power line is required to reach each module, and then the integrated valves regulate it to the specific fluidic actuator, as in [25]. This approach means that fluidic powering is no longer dependent on the total amount of modules, however the electronic control of all the valves still requires a similar number of controlling lines. Therefore, the resulting dependence of $N_{L}$ over $N_{M}$ ad $N_{A}$ is not changed and is also increased by one line due the single power fluidic line running through the whole system (Fig. 3).

To make the modular system completely independent of $N_{M}$ and $N_{A}$, we propose a modified integrated control, the wireless integrated control. Thanks to the wireless technology the control of the integrated valves can be transferred by means of wireless signals from the central systems to the modules. In this control, $N_{L}$ is not dependent on $N_{M}$ (Fig. 3) as there are only two main power (fluidic and control) lines in the system.

The architecture of this system is shown in Fig. 4, with the two power lines depicted in in blue (fluidic) and in red (electric). The control logic is managed by a dongle connected to the master system (central system), which wirelessly transmits the controlled state of each valve to the corresponding module. Each module is equipped with a wireless board, which provides all the electronic components for actuating the module depending on the control signals. The system includes a pressure regulator which is positioned externally from the modular robot and is used for the proportional pressure regulation of the main fluidic line $\left(P_{\text {line }}\right.$ in Fig. 4), supplied to all the valves inlets. Given that, the inlets are already pressurecontrolled means that highly miniaturized two-way operation valves can be integrated inside each module without compromising the module's size and weight. These simple valves act as fluidic gates which connect (when open) the corresponding fluidic actuator to the pressure value of the main fluidic line $\left(P_{\text {line }}\right)$.

Therefore, by using a temporized control of all the valves and $P_{\text {line }}$, the central system can manage both the inflation and the deflation of all actuators (chambers) of the system. The inverse of this temporization is the frequency of the low-level control, which allows to address the pressure in one actuator at a time. So the pressure inside the $\mathrm{j}$-th actuator in the $\mathrm{i}$-module $\left(P_{i j}\right)$ can be expressed as:

$$
\begin{cases}P_{i j}(t)=P_{\text {line }}(t), & \text { valve open } \\ P_{C_{i j}}(t)=P_{\text {line }}\left(t_{i j c l o s i n g}\right), & \text { valve closed }\end{cases}
$$

where $t_{i j c l o s i n g}$ is the instant in time when the valve $V_{i j}$ switches its state from open to closed. Thus $P_{i j}$ stays constant at this value until the valve re-opens and $P_{i j}$ starts to follow again the $P_{\text {line }}$.

Globally, to control the assembled manipulator an high level shared time control (i.e. periodic control) is needed.

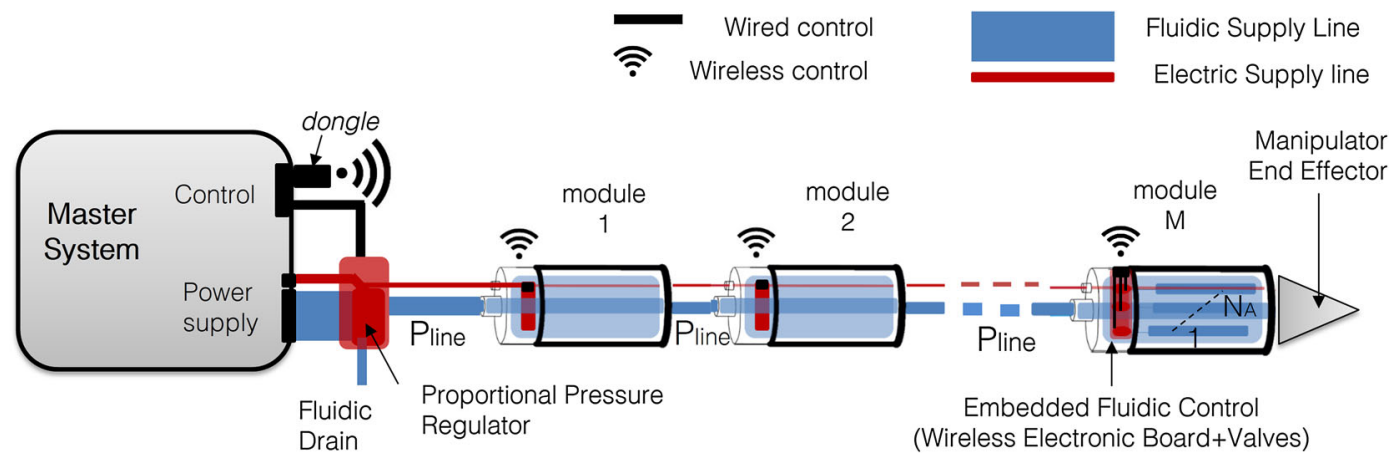

Fig. 4 System architecture of the soft modular manipulator 
This control strategy, if, from one side, makes possible the implementation of a complete modular architecture, on the other side it involves two critical issues. Firstly, with this architecture, it is not possible to contemporary inflate two chambers with different pressure. This presents limitations in terms of trajectories that involve the actuation of more than one chamber at a time. Secondly, if one looks at the global control, it results that the control frequency of the assembled manipulator (the high-level control) is affected by the number of modules and, in the worst case, is decreased by a factor of $1 / N_{M} N_{A}$. This means that the maximum achievable dynamics of the manipulator is potentially slowed down by increasing number of modules.

However, both these problems can be mitigated by increasing, as much as the possible, the previously mentioned low-level control frequency. In fact, the higher is this frequency, the less appreciable would be the effects of both these issues: (1) the trajectories, involving the actuation of more than one chambers, can be finely approximated by periodic small steps of pressure in the chambers (lowering the maximum achievable velocity of the trajectories movements but preserving all the workspace reachable); (2) the dynamics of the system is enhanced, partially compensating for the effect of the number of modules on the high-level control frequency of the whole manipulator.

In addition, since surgical devices are generally required to be maneuvered at very low speed (around $1 \mathrm{~Hz}$; [5]), the central control has enough time to make the manipulator following the required trajectories with such a control strategy. Moreover, considering that the available workspace for the assembled manipulator in MIS is actually limited to a volume comparable an inflated human abdomen (at maximum), the number of required modules would never be too large to cause a relevant reduction of the high-level control frequency of assembled manipulator.

Finally, it is worth noting that the ability to enhance the low-level control frequency is directly dependent on the specification of the hardware components of the system, such as the millions of instruction per second (named MIPS) of the microcontroller, the wireless transmission rate and the operating time of the valves.

\section{Design, fabrication and assembly of the integrated module}

This section presents the design and fabrication of a fully integrated module according to the scheme in Fig. 4.

The soft actuator is a 3DOF cylindrical FFA, which incorporates three expandable chambers equally arranged around the main central axis $\left(N_{A}=3\right)$. They are essentially three empty chambers created in a cylindrical bulk of elastomeric material, which can be pressurized either alternately to bend the cylinder or simultaneously to elongate it [26-28]. Cylindric 3DOF FFAs need to limit the severe lateral expansion (i.e. ballooning effect), which inevitably happens at the external side of the chambers when inflated, due to the thin wall of the elastomeric material, as reported in most of the works employing FFAs. Limiting this expansion would not only make the actuation safer, thus avoiding the risk of explosion, but would also enhance the FFA's performance, in terms of maximum bending angle and regularity of the curvature radius.

Various strategies have been proposed to tackle this problem and mainly regard the addition of reinforcement elements around or embedded in the elastomeric material [29, 30]. The approach here is to use an external bellow-shaped sheath which, as demonstrated in [31], limits the lateral expansion of the elastomer underneath and enhances the response of the actuator to the input pressure. The pictures in Fig. 5 highlights the difference between an FFA without any elastomer expansion retain mechanism (Fig. 5a), and one with an external sheath (Fig. 5c).

With the retain mechanism, the effect of the chamber insufflation is entirely transformed into the motion of the module, while in the pure elastomeric structure, part of the pressurization is spent and lost inside the external expansion chamber. A vascular graft was selected to act as a retain sheath, since it is bellow-shaped and with the appropriate size and flexibility. Vascular grafts are bellow-warped knitted polyester prostheses which are conventionally used to replace a piece of vascular tract. Their proprieties match perfectly with the requirements for a retaining sheath to contain the elastomer ballooning effect of FFAs, as they are dilatant-resistant but, at the same time, they can be bent, elongated and squeezed with minimal stress. A vascular graft is also a certified and 




Fig. 5 a FFA actuator without retain mechanism; b flow diagram of the fabrication of the FFA actuator; $\mathbf{c}$ FFA actuator with retain mechanism; d FFA's mold exploded parts and employed vascular graft

biocompatible medical device, thus adequate for potential contacts with internal organs.

The fabrication of the FFA, as shown by the flow diagram in Fig. 5b, starts with the production of the elastomeric body $(9 \mathrm{~mm}$ in diameter and $30 \mathrm{~mm}$ in length), and then the sheath is added (10 $\mathrm{mm}$ is the actual module external diameter). First, the inner body is fabricated by casting uncured silicone (EcoFlex 0050, Smooth-On, Inc., USA) [32] into a cylindrical mold containing the positive shape of the chambers and channels in order to create the empty spaces in the silicone. The mold is an assembly of different components (Fig. 5d): an external cylindrical shell in Delrin and laser cut (Universal Laser VLS 2.30) plexiglass plates for aligning the 3D printed (Invision Si2, 3D Systems, Inc., UK) chamber molds at the base and top of the shell mold during the silicone curing. Once poured into this mold, the silicone is stabilized at room temperature for $2 \mathrm{~h}$ and then cured at $60^{\circ} \mathrm{C}$ for $30 \mathrm{~min}$. When removed from the mold, the silicone body is used as a mold itself to pour a higher tensile strength silicone (Dragonskin 30, Smooth- On, Inc.,
USA) [33], which acts as a spine of the module and as a pipe to carry the fluidic power towards the modules top side, where it is available for another module. For the characterization of the material, see [34], where tests according to ISO37:2005(E) are reported in detail for all the silicones used in the FFA. Finally, the retaining sheath (a knitted polyester vascular graft produced by Vaskutek Inc., Terumo Company, UK [35]) is slid around the silicone body and fixed at its top and bottom extremities (Fig. 5c).

As previously mentioned, a two-way operation latching solenoid valve is connected at each chamber, as reported in Fig. 6. The chosen valves (LF solenoid valves, series 120 , by Lee Products Ltd, UK) are the most miniaturized (3 $\mathrm{mm}$ in diameter) and light weight $(300 \mathrm{mg})$ solenoid valves available on the market. Thanks to their magnetically bi-stable (latching) mechanism, they need power just for switching their state (from closed to open, and vice versa) with a power consumption of about $1.8 \mathrm{~mW}$ s/switch (the switching requires a $1 \mathrm{~ms}$ pulse of about $5 \mathrm{VDC}$ ). To provide the control signals to the valves and to communicate wirelessly with the central system, a double-sided PCB electronic board was developed. The PCB board (Fig. 6) is $10 \mathrm{~mm}$ in diameter and incorporates a wireless microcontroller (CC2430, Texas Instruments, USA) embedding a telemetry communication system (wireless controllability over 2.4 GHz, ZigBee compliant IEEE 802.15.4 transceiver), a voltage regulator, and drivers for the valves (A3901, Allegro Microsystems LLC, USA). The PCB board communicates wirelessly with the dongle connected to the master control system (Fig. 4), which incorporates another CC2430-based board. This module communicates with the control PC through an off-

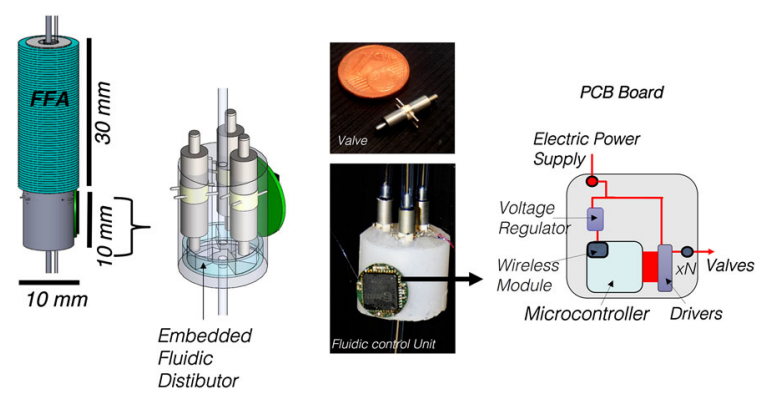

Fig. 6 From left to right: Integrated module design, CAD view of the actuation unit, pictures of the employed valves and assembled actuation unit, scheme of PCB electronic board 
the-shelf USB/serial universal asynchronous receivertransmitter (UART) and a converter development module for the FT232R IC device (UM232R, Future Technology Devices International Ltd, UK). Through this dongle board, the master system also controls the external proportional pressure regulator (proportional pressure micro-regulator, series K8P, Camozzi Group, IT).

To respect the modularity specification, all modules should have uniform interfaces. As each module contains three fluidic actuators, a fluidic power distribution point is required inside the module itself. This distribution point can be designed as a fluidic chamber with five branches: one branch for the single inlet (the main fluidic line) and $1+N_{A}$ branches for the outlets (the $N_{A}$ valves inlets and the continuing fluidic line for the next module). Although commercial solutions offer several options for such a multi-way connector, but none of them fitted in the current module design, because of their shape/size or their material stiffness. For this reason, a specifically customized multi-way fluidic distributor was designed, optimized and then integrated into the module.

The fluidic distributor can take different shapes depending on the position of the inlet and the outlets. In this work the geometry of the fluidic distributor (five-way) is represented by a Y-like empty space inside the elastomeric material, as depicted in Fig. 7c, which can be reached by the inlet pipe at one side, and by the four outlets (three of them equally organized around the center) on the other side.

Unlike the FFA's chambers, the fluid pressure must not cause a minimal expansion in the elastomeric bulk of material. Therefore the fluidic distributor needs to be designed to keep its initial shape even when it has the pressurized fluid inside. A preliminary (finite elements method (FEM) analysis, operated in SIMULIA ABAQUS (Dessault Systems, USA), was performed before the actual fabrication of the elastomeric connector. The aim of the FEM study was to understand if the material of the distributor was able to sustain the range of expected working pressures, without producing considerable deformation in the elastomer. The simulation was performed on a distributor made up of a silicone with a higher tensile strength than the one in the FFA, i.e the DragonSkin 30 (by Smooth-On Inc., USA), using a dynamic implicit analysis and applying a tetrahedral mesh with quadratic order elements to the elastomeric structure. The simulated pressures supplied to the branched chamber inside the silicone fluidic connector ranged from 0 up to $70 \mathrm{kPa}(0-10 \mathrm{psi})$, which is twice the maximum working pressure range of the FFA. A peak of $180 \mathrm{kPa}$ was also imposed to detect if considerable deformations of the distributor would happen in case of possible over-pressurization due to a failure of the control system. This FEM analysis, (Fig. 7a, b, d) confirmed that the distributor would experience negligible deformations (less than $6 \%$ in radius expansion)within the FFA working pressure range. However, for higher pressures (more than $70 \mathrm{kPa}$ ) the deformation of the structure begins to be noticeable (over $10 \%$ ) and is mainly located at the lateral border of the distributing chamber, where the silicone wall is thinner (as highlighted in Fig. 7d). According to these preliminary simulation results, the design of the actual fluidic distributor incorporates a reinforcement layer in this area. This reinforcement is provided by the addition of an even higher tensile strength silicone, the Smooth Sil 950 (by Smooth-On Inc, USA).

The fabrication of our elastomeric fluidic distributor involves the production of the elastomeric body, again, by casting silicones (DragonSkin 30 for the main part and Smooth Sil 950 for the reinforcing layer). In this case the molds are assembled from laser cut plexiglass sheets ( $2 \mathrm{~mm}$ in thickness) (Fig. 8). The fabrication is depicted in Fig. 8, which shows the steps to build an empty branched chamber inside the silicone bulk and make it accessible for the inlet/ outlet pipes and valves. The two sides of the fluidic distributor, depicted in green and blue in Fig. 8, are first cast separately using the respective mold (step 1). Then they are hermetically sealed by pouring another layer of uncured silicone around the two merged sides (step 2). This strategy facilitates the integration of the reinforced layer, which can be attached to the outlet side (see the picture in the inset in Fig. 8), by adding a silicone casting Smooth Sil 950 to the outlet side, using the outlet side mold, before casting and filling up the same mold with DragonSkin 30.

\section{Test methodology}

The functionality of the fully-integrated module is above all related to the correct operation of the 
(a)

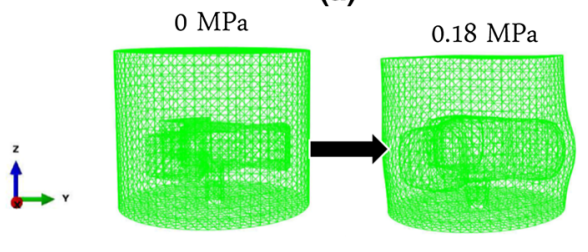

Distributing (c) chamber

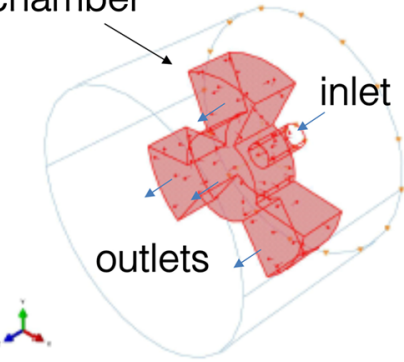

Fig. 7 FEM simulation of the expansion produced by the applied pressure to the wall of the distributing chamber of fluidic connector. The images report the null deformation phase (0 MPa) and the maximum deformation point $(0.183 \mathrm{MPa})$. a Initial and deformed shape of the distributing chamber (mesh

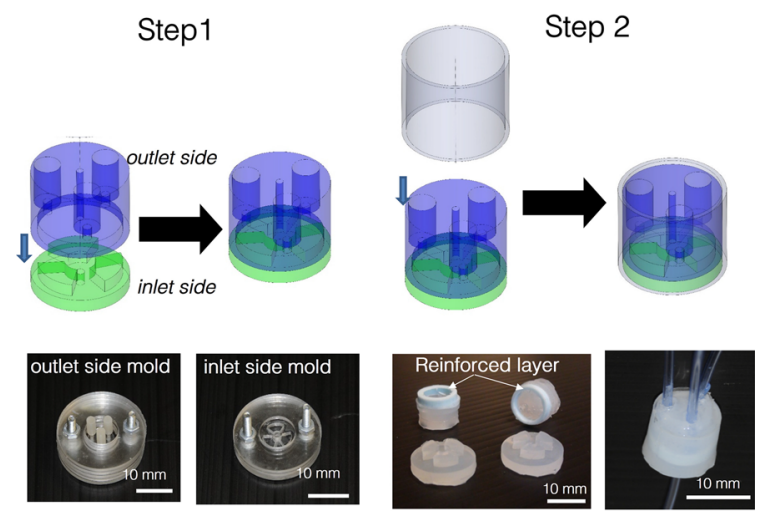

Fig. 8 Step 1 The two parts of the silicone distributor are separately casted in the respective mold and then merged. Step 2 The two silicone parts are merged and sealed by pouring and casting another layer of silicone externally

custom-made fluidic distributor and to the module performance in response to the wireless control signals. The fluidic distributor needs to be assessed in terms of pressure drops caused by the passage of the fluid through the system. This means calculating the fluidic resistance offered by the system and comparing it with the resistance of a rigid and standard connector

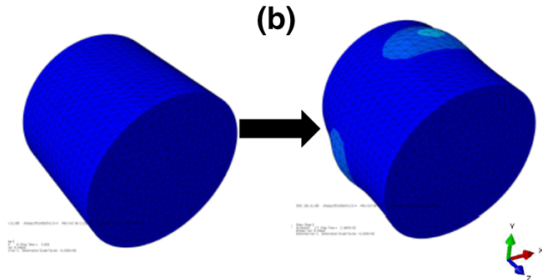

(d)



view); $\mathbf{b}$ initial and deformed shape of the distributing chamber (post-processing data); c distributing chamber; d deformed shape of the distributing chamber, section view (post processing data)

of a similar size (in lumen diameter) in order to verify its reliability.

The system was thus analyzed by exploiting the equivalence between the fluidic and the electrical domain [36]. According to this equivalence, the fluid pressure, the flow, the pipes/connectors and the fluidic actuators (i.e the expandable FFA's chambers) of the fluidic circuit become the voltage, current, the resistance and the capacitances of an equivalent electric circuit, respectively. This method is called lumped parameter modeling and dramatically simplifies the modeling of complex and continuous systems. Therefore, a fluidic circuit presenting distributed parameters can be studied using its equivalent lumped parameter electrical model. The fluidic resistance, like an electrical resistor, determines a drop in pressure (voltage potential) with the passage of fluid (current), which cannot be recovered because it is dissipated through irreversible processes (such as heat and friction). Pipes, tubes and connectors can be considered fluidic resistances, because they cause a pressure drop, this is due their geometrical parameters, their materials and the viscosity of the fluid passing through them. It is thus important to evaluate the actual resistance offered by the fluidic distributor in order to 
quantify the pressure drop caused by its inner channeling system.

The equivalent circuits of Fig. 9 were therefore considered. The test considers two pressure measurements $\left(P_{1}\right.$ and $\left.P_{2}\right)$, which are taken first in circuit 1 $\left(P_{1}^{\prime}, P_{2}^{\prime}\right)$ and then in circuit $2\left(P_{1}^{\prime \prime}, P_{2}^{\prime \prime}\right)$. The two systems differ in terms of the resistance, which is placed in between the measurements points, while $R_{K}$ is the same in both circuits and corresponds to the resistance of pipe $\mathrm{K}$ which is kept in the same place (between the pressure sensor $P 2$ and the drain to the atmospheric pressure) during the pressure measurements in the two circuits. In circuit $1, R_{T}$ is known, while in circuit 2 this resistance is replaced by the system whose fluidic resistance $\left(R_{X}\right)$ needs to be evaluated (the connector/ distributor). The reference resistance $R_{T}$ is a pipe of known length (L) and inner diameter (D), which can be expressed as:

$$
R_{T}=\frac{8 \mu L}{\pi\left(\frac{D}{2}\right)^{4}}
$$

where $\mu$ is the viscosity of the fluid. Therefore, by measuring the pressures in the first circuit $P_{1}^{\prime}$ and $P_{2}^{\prime}$ then in the second circuit $P_{1}^{\prime \prime}$ and $P_{2}^{\prime \prime}$, the following equations can be derived:

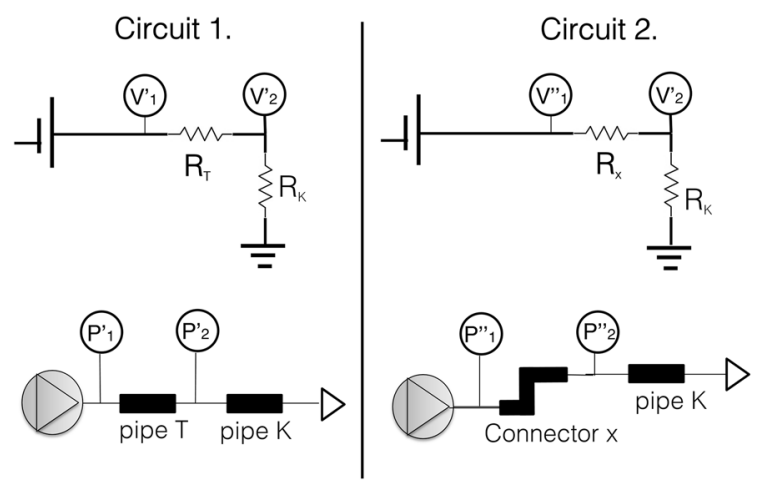

Fig. 9 Circuits for the experimental evaluation of the distributors fluidic resistance (top: scheme of the equivalent electric circuit, bottom: scheme of the real fluidic circuit). The circuit 1 is used to determine $R_{K}$ for the test on the circuit 2. During the test the connector $\mathrm{x}$ has been replaced by the developed distributor connectors and the reference commercial connector. For the fluidic distributors (with 3 outlets), $P_{2}^{\prime \prime}$ was measured at one of its outlet at a time while closing the others

$$
\left\{\begin{array}{l}
\frac{P_{1}^{\prime}-P_{2}^{\prime}}{R_{T}}=\frac{P_{2}^{\prime}}{R_{K}} \\
\frac{P_{1}^{\prime \prime}-P_{2}^{\prime \prime}}{R_{X}}=\frac{P_{2}^{\prime \prime}}{R_{K}}
\end{array}\right.
$$

The resistance $R_{K}$ only serves to maintain the flow active during the pressure acquisition time and it is represented by a longer and narrower pipe than $R_{T}$. From (3), the unknown resistance $R_{X}$ can be expressed as:

$R_{X}=\frac{P_{1}^{\prime \prime}-P_{2}^{\prime \prime}}{P_{2}^{\prime \prime}} \frac{P_{2}^{\prime}}{P_{1}^{\prime}-P_{2}^{\prime}} R_{T}$

Once the resistance $R_{K}$ had been calculated, the tests made use of just circuit 2 to evaluate the resistance $R_{X}$ corresponding to the connectors placed in between $P_{1}^{\prime \prime}$ and $P_{2}^{\prime \prime}$. The test was performed on three types of fluidic distributors: (1) made up of solely DragonSkin30; (2) on fluidic distributors with the embedded reinforcement layer in Smooth Sil 950; and (3) on a reference $90^{\circ}$ elbow barbed connector made up of rigid plastic ( $1 / 16^{\prime \prime}$ fitting (by Cole-Parmer Chemicals, USA) which offers a similar path for the fluid to that of the distributor.

The module motion performances were experimentally validated to estimate the actuators behavior in response to the pressure, thus allowing a preliminary open loop control of the system. The set up used for this test is reported in Fig. 10. The module was secured at its root with a hollow frame, which enabled the fluidic actuation unit underneath to be connected. In order to track the displacement of the module tip in the space, an electromagnetic tracking system (Aurora, Northern Digital, Inc., NDI) was used. The Aurora system includes a small probe (Aurora Mini 6DOF Sensor, $1.8 \times 9 \mathrm{~mm}$ ), secured at the top of the module, whose movements are detected by the Auroras magnetic field generator which covers the workspace area. This system was chosen for its high accuracy (RMS: $0.8 \mathrm{~mm}$ in position, 0.70 in orientation) and working frequency $(40 \mathrm{~Hz})$ [37]. Its acquisition was synchronized with the signal obtained from a pressure sensor (26PC SERIES, Honeywell), which was placed in between the valve outlet and the chamber.

This test rig was used to assess the static and dynamic characteristics of the actuator for one basic motion (i.e the one-chamber bending) and to determine the entire workspace of a single module. For the 
Fig. 10 Scheme of the test rig for the static and dynamic performances of the module and picture of the real set up

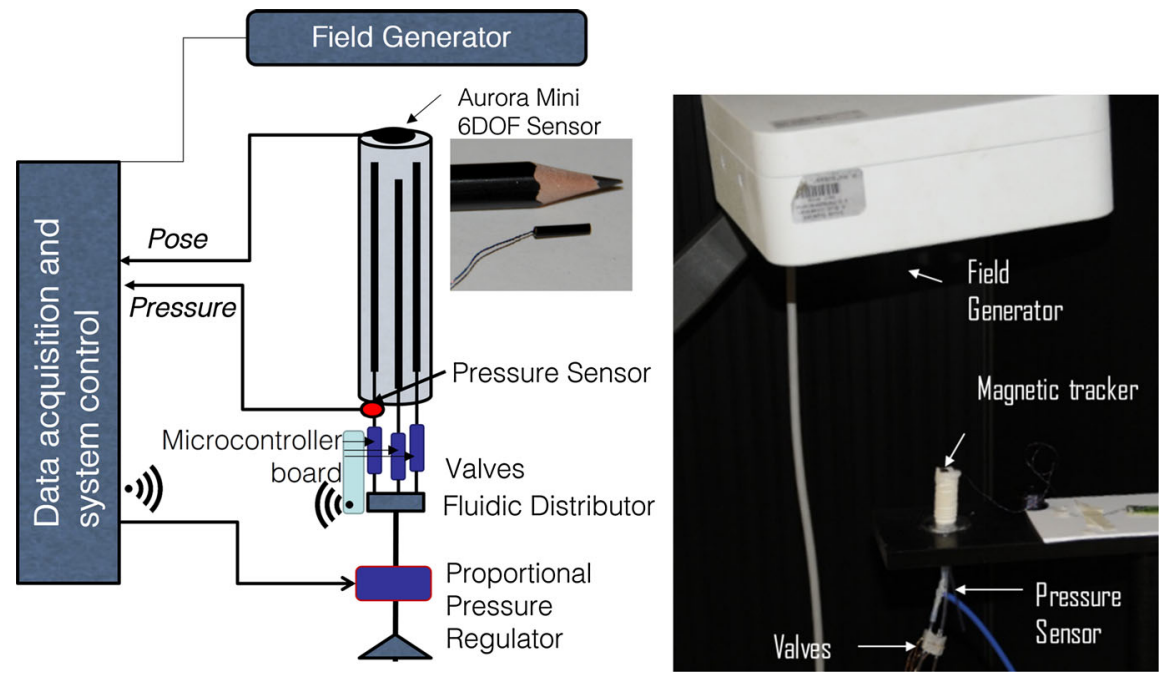

static test and workspace evaluation, the chambers were inflated in a range of $0-5 \mathrm{psi}(0-34.47 \mathrm{kPa})$, with regularly imposed steps of $0.1 \mathrm{psi}$. The dynamic tests consisted of tracking the tip motion over time in response to discrete steps of input pressure in order to analyze its dynamic behavior. This input was produced by setting the pressure line at the step value and then opening the related valve.

The electric equivalence of an entire module was thus used as a reference to explain the response of a module's chamber to a pressure input step (Fig. 11). The input pressure $u(t)$ corresponds to the pressure measured when the valve $V_{i j}$ is closed by the wireless signal, and consequently excites the ij-th chamber. The ij-th chamber is made up of a distributor branch, a valve and a fluidic inflatable chamber, which produces

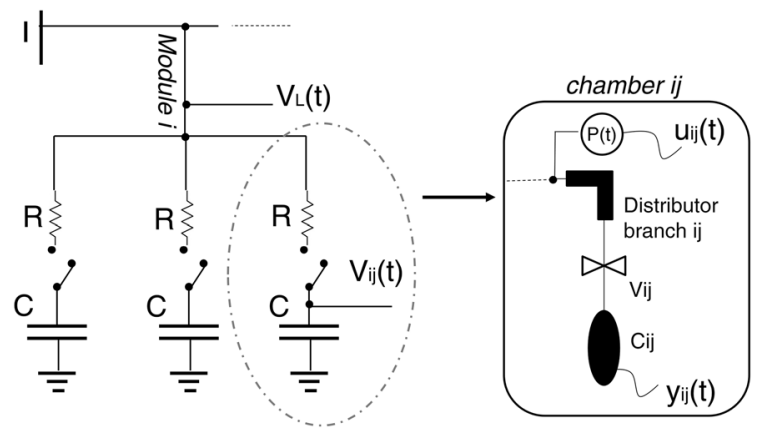

Fig. 11 Equivalent electric circuit for the single module i(inset: fluidic circuit of the system chamber $i j, u_{i j}$ is the input pressure and $y_{i j}$ is the displacements, in terms of bending angle, of the module $\mathrm{i}$ due to the inflation of the chamber ij the related module's motion if pressurized. Measuring the tracked module displacement would result in determining the output of the system $y(t)$, which in Fig. 11 corresponds to measuring the voltage over the capacitance. Once the steps, input $u$ and output $y$ in four points of the static characteristic, were acquired, a parameter identification analysis was performed by the Matlab Identification ToolBox (The MathWorks, Inc.)

\section{Results and discussion}

Figure 12 reports the mean values of the fluidic resistance tests obtained for the fluidic distributors (normal and reinforced) and the commercial $90^{\circ}$

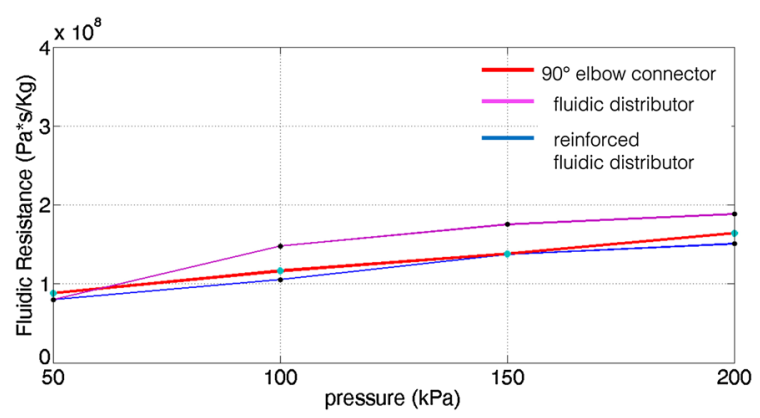

Fig. 12 Experimental fluidic resistance. Three prototypes for fluidic distributors (with and without reinforced layer) were analyzed, while for the $90^{\circ}$ rigid elbow the measures on the circuit 2 were repeated three times for statistics 
elbow connector, chosen for comparison. The graph shows that the resistance offered by the custom-made reinforced distributor is comparable with the resistance of a standard and rigid $90^{\circ}$ elbow connector, which was measured by using the same circuit as in Fig. 9.

The possibility of obtaining a reliable channeling system inside the bulk of the elastomeric material paves the way for the use of custom connectors/ distributors which can adapt to the various sizes and geometry of the related FFAs, without compromising the overall softness and compliance of the system.

The static tests on the module performing one chamber bending motion are reported in Fig. 12. The bending trajectory describes the displacement of the module tip in the plane perpendicular to the module base and passing through the center of the inflated chamber. Both the inflating and deflating curves describe an arc of circumference, which deviates from the ideal case with a maximum error of $2.44 \%$. The static characteristic with the span angle $(\theta)$ described by the module's tip on these trajectories in response to the input pressure is shown in Fig. 13(right). The sigmoid curves and hysteresis are representative of the relevant non-linearity of the system as an assembly of hyper-elastic materials.

Figure 14 shows the workspace covered by the single module. The module elongation capability (up to $70 \%$ ) is depicted in black (Fig. 14a). The isotropic behavior of the module is clearly visible in the plot in Fig. 14b (top view of the workspace), as the inflation of each chamber makes the module follow similar and equally spaced trajectories around the center trajectories.
The dynamic behavior of the actuator was investigated by exciting the system with step inputs generated by the wireless controlled valves. Using the respective experimental data of the step of input pressure $u(t)$ and the tracked output displacement $y(t)$, which is the span angle $(\Theta)$ in the case of one chamber bending, a parameter identification analysis was performed. Four significant values of the static inflation characteristic (highlighted in Fig. 3) of the one chamber bending motion, defined as equilibrium points (EP), were selected. These points were used to excite the system with step inputs and obtain the data for the system identification analysis. To cover all the potential responses that the model could show (i.e. delays, oscillations due to the presence complex conjugate poles, possible interaction between two close positioned poles), the identification was carried out by starting with a second order system with delay. Nevertheless, the identification analysis demonstrated that the model of the system fits into a first order model without delay, whose transfer function is:

$G(s)=\frac{Y(s)}{U(s)}=\frac{1}{1+s T}$

and the time response is expressed as:

$y(t)=K\left(1-e^{\frac{-t}{\tau}}\right) u(t)$

In fact, between the two identified real poles, one pole (T) was found to be five orders of magnitude greater than the other pole, which can therefore be neglected, because the resulted delay, due to this pole, was small enough to be comparable with the timing of the data acquisition system. The same model
Fig. 13 a One plane trajectory in the case of one chambers being inflated/ deflated from 0 to 5 psi $(0-$ $34.7 \mathrm{kPa}$ ); b Static characteristic of the output span angle $(\theta)$ versus the input pressure. Four equilibrium points $(\mathrm{EP} 1=3.8$ psi, $\mathrm{EP} 2=4.2 \mathrm{psi}$, $\mathrm{EP} 3=4.8 \mathrm{psi}$, $\mathrm{EP} 4=5$ psi) have been considered for the dynamic analysis
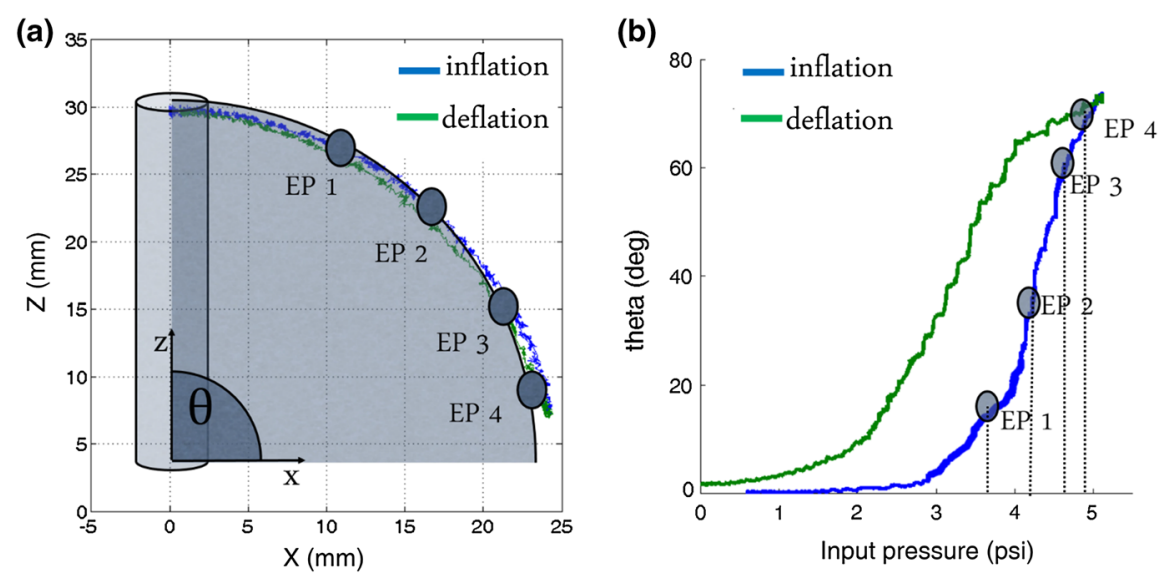
Fig. 14 a $3 D$ view of the reachable workspace of the single module. The trajectories of the single chamber inflation motions are shown in blue, red and purple. $\mathbf{b}$ Top view of the workspace. (Color figure online)

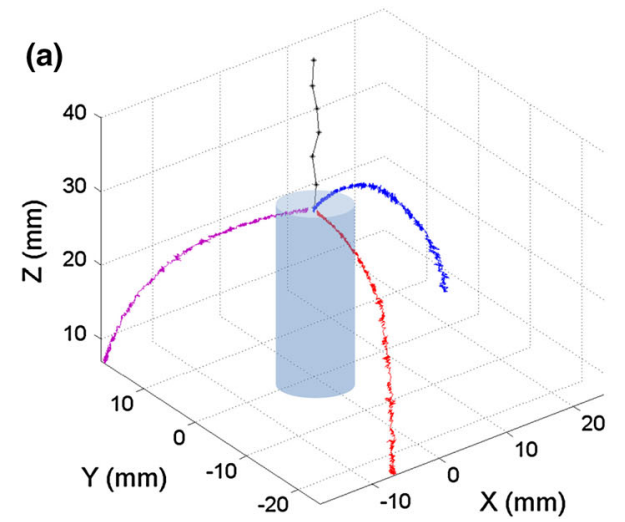

configuration (first order with no delays) was found to be valid in each equilibrium point, all showing a single dominant pole (T) and a correctly scaled static gain $(\mathrm{K})$, thus supporting the reliability of the model in describing the module's behavior (Fig. 15a). To verify the parameter estimation of the model, the nominal output of the model was compared with the experimental data outputs. The comparison between the model and the experimental outputs on the last equilibrium point is reported in Fig. 14c. Despite the nonlinearity and visco-elasticity, which inevitably affect the whole system, the dynamic behavior of the single module can be greatly simplified by considering it as a first order system with a linear behavior (with a proper static gain). This demonstrates that the module

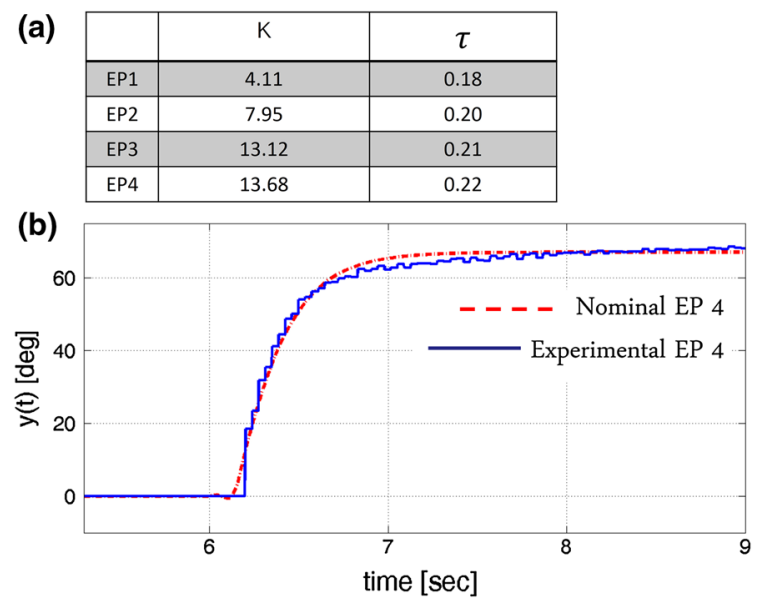

Fig. 15 a Table with the parameter estimation results, the static gain $\mathrm{K}$ and the pole value $\mathrm{T}$ about the four analyzed equilibrium points. b Comparison between the nominal and the experimental output for the case of equilibrium point (EP 4), as the worst case scenario in terms of time constant

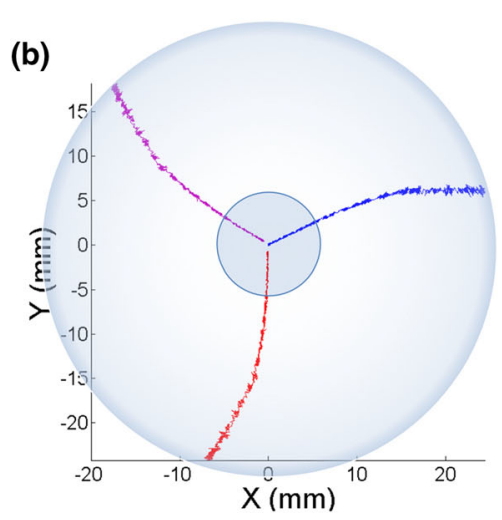

can be reasonably compared to the electrical circuit in Fig. 11 in terms of dynamic behavior, thus potentially simplfying the control of the whole manipulator.

\section{Conclusions}

This paper presents the key technology for building a fully modular soft manipulator, which exploits flexible fluidic actuators for use in minimally invasive surgery. This preliminary study can be considered as a starting point for the future implementation and control of a multi-modular manipulator whose length is not defined a priori. It would therefore be possible to assemble it in different configurations depending on the specific surgical task to be performed, as depicted in Fig. 1. Our strategy is based on the use of wireless controlled modules, which all interface with a single fluidic-control line with the rest of the system. In this way, the manipulator can be configured depending on the needs of the specific surgical operation. The aim of the paper was focused on the development and complete characterization of the single fully integrated soft module, as a key part of the modular structure. Each module is equipped with an FFA with three chamber-fluidic-actuators, three miniaturized latching valves, a wireless microcontroller board and a specifically designed fluidic distributor integrated in the elastomeric material that constitutes the whole module. The entire mobility of the whole module was evaluated in terms of workspace and static characteristic. From the parameter estimation analysis, the module's dynamic model during one-chamber motion was estimated. The aim of the full characterization of this single module was to facilitate the future high 
level control of the multi-modular architecture of the MIS manipulator. Future developments regard the further improvement of the single module, for example by integrating a bending sensor for each chamber and easily readable by the PCB integrated wireless microcontroller, as well as an appropriate end-effector module at the tip to really accomplish surgical tasks.

Acknowledgments This work has been partially supported by the European Commission with the STIFF-FLOP IP (287728) and the ROBOSOFT CA (619319).

Open Access This article is distributed under the terms of the Creative Commons Attribution 4.0 International License (http:// creativecommons.org/licenses/by/4.0/), which permits unrestricted use, distribution, and reproduction in any medium, provided you give appropriate credit to the original author(s) and the source, provide a link to the Creative Commons license, and indicate if changes were made.

\section{References}

1. Vitiello V, Lee S-L, Cundy TP, Yang G-Z (2013) Emerging robotic platforms for minimally invasive surgery. IEEE Rev Biomed Eng 6:111-126

2. Marescaux J, Dallemagne B, Perretta S, Wattiez A, Mutter D, Coumaros D (2007) Surgery without scars: report of transluminal cholecystectomy in a human being. Arch Surg 142(9):823-826

3. Andrea B, Dharamsi Latif M, Netterville James L, Gaelyn GC, Nabil S (2013) Robotic-assisted micro-surgery of the throat: the trans-nasal approach. In 2013 IEEE international conference on robotics and automation (ICRA), IEEE, pp 232-238

4. Cowan Lara S, Walker Ian D (2013) The importance of continuous and discrete elements in continuum robots. Int $\mathbf{J}$ Adv Robot Syst 10(165). doi:10.5772/55270

5. Degani A, Choset H, Zubiate B, Ota T, Zenati M (2008) Highly articulated robotic probe for minimally invasive surgery. In: Engineering in Medicine and Biology Society, 2008. EMBS 2008. 30th annual international conference of the IEEE, pp 3273-3276

6. McMahan W, Chitrakaran V, Csencsits M, Dawson D, Walker Ian D, Jones Bryan A, Pritts M, Dienno D, Grissom M, Rahn Christopher D (2006) Field trials and testing of the octarm continuum manipulator. In: Proceedings 2006 IEEE international conference on robotics and automation, 2006. ICRA 2006, IEEE, pp 2336-2341

7. Yim M, White P, Park M, Sastra J (2009) Modular selfreconfigurable robots. In: Meyers RA (ed) Encyclopedia of complexity and systems science. Springer, New York, pp 5618-5631

8. Russo S, Harada K, Ranzani T, Manfredi L, Stefanini C, Menciassi A, Dario P (2013) Design of a robotic module for autonomous exploration and multimode locomotion. IEEE/ ASME Trans Mechatron 18(6):1757-1766
9. Salvietti G, Prattichizzo D, Niccolini M, Ranzani T, Menciassi A (2012) A task priority approach for modular robots in minimally invasive surgery. In: ICRA 2012 workshop: modular surgical robotics: how can we make it possible?

10. Harada K, Oetomo D, Susilo E, Menciassi A, Daney D, Merlet J-P, Dario P (2010) A reconfigurable modular robotic endoluminal surgical system: vision and preliminary results. Robotica 28(02):171-183

11. Tortora G, Dario P, Menciassi A (2014) Array of robots augmenting the kinematics of endocavitary surgery. IEEE/ ASME transactions on mechatronics

12. Majidi C (2014) Soft robotics: a perspective-current trends and prospects for the future. Soft Robot 1(1):5-11

13. Laschi C, Cianchetti M (2014) Soft robotics: new perspectives for robot bodyware and control. Bion Biomim 2:3

14. Ilievski F, Mazzeo AD, Shepherd RF, Chen X, Whitesides GM (2011) Soft robotics for chemists. Angewandte Chemie 123(8):1930-1935

15. Albu-Schaffer A, Eiberger O, Grebenstein M, Haddadin S, Ott C, Wimbock T, Wolf S, Hirzinger G (2008) Soft robotics. IEEE Robot Autom Mag 15(3):20-30

16. Hirzinger G, Albu-Schaffer A, Hahnle M, Schaefer I, Sporer N (2001) On a new generation of torque controlled lightweight robots. In: Proceedings 2001 ICRA. IEEE international conference on robotics and automation, 2001. vol 4, IEEE, pp 3356-3363

17. Cianchetti M, Ranzani T, Gerboni G, Nanayakkara T, Althoefer K, Dasgupta P, Menciassi A (2014) Soft robotics technologies to address shortcomings in today's minimally invasive surgery: the stiff-flop approach. Soft Robot 1(2):122-131

18. Ranzani T, Gerboni G, Cianchetti M, Menciassi A (2014) A bioinspired soft manipulator for minimally invasive surgery. Bioinspir Biomim 10(3). doi:10.1088/1748-3190/10/ 3/035008

19. De Greef A, Lambert P, Delchambre A (2009) Towards flexible medical instruments: review of flexible fluidic actuators. Precis Eng 33(4):311-321

20. Piccigallo M, Scarfogliero U, Quaglia C, Petroni G, Valdastri P, Menciassi A, Dario P (2010) Design of a novel bimanual robotic system for single-port laparoscopy. IEEE/ ASME Trans Mechatron 15(6):871-878

21. Petroni G, Niccolini M, Menciassi A, Dario P, Cuschieri A (2013) A novel intracorporeal assembling robotic system for single-port laparoscopic surgery. Surg Endosc 27(2):665-670

22. Laschi C, Cianchetti M, Mazzolai B, Margheri L, Follador M, Dario P (2012) Soft robot arm inspired by the octopus. Adv Robot 26(7):709-727

23. Fu Y, Harvey EC, Ghantasala MK, Spinks GM (2006) Design, fabrication and testing of piezoelectric polymer PVDF microactuators. Smart Mater Struct 15(1):S141

24. Shepherd RF, Ilievski F, Choi W, Morin SA, Stokes AA, Mazzeo AD, Chen X, Wang M, Whitesides GM (2011) Multigait soft robot. Proc Natl Acad Sci USA 108(51): 20400-20403

25. Onal CD, Rus D (2013) Autonomous undulatory serpentine locomotion utilizing body dynamics of a fluidic soft robot. Bioinspir Biomim 8(2):026003

26. Suzumori K, Iikura S, Tanaka H (1992) Applying a flexible microactuator to robotic mechanisms. IEEE Control Syst 12(1):21-27 
27. Chang B, Chew A, Naghshineh N, Menon C (2012) A spatial bending fluidic actuator: fabrication and quasi-static characteristics. Smart Mater Struct 21(4):045008

28. Martinez RV, Branch JL, Fish CR, Jin L, Shepherd RF, Nunes R, Suo Z, Whitesides GM (2013) Robotic tentacles with three-dimensional mobility based on flexible elastomers. Adv Mater 25(2):205-212

29. Galloway Kevin C, Polygerinos P, Walsh Conor J, Wood Robert J (2013) Mechanically programmable bend radius for fiber-reinforced soft actuators. In: 2013 16th international conference on advanced robotics (ICAR), IEEE, pp 1-6

30. Shapiro Y, Wolf A, Gabor K (2011) Bi-bellows: pneumatic bending actuator. Sens Actuators A Phys 167(2):484-494

31. Cianchetti M, Ranzani T, Gerboni G, De Falco I, Laschi C, Menciassi A (2013) Stiff-flop surgical manipulator: mechanical design and experimental characterization of the single module. In: 2013 IEEE/RSJ international conference on intelligent robots and systems (IROS), IEEE, pp 3576-3581

32. http://www.smooth-on.com/tb/files/ECOFLEX_SERIES_ TB.pdf

33. http://www.smooth-on.com/tb/files/DRAGON_SKIN_SERI ES_TB.pdf

34. Elsayed Y, Lekakou C, Ranzani T, Cianchetti M, Morino M, Arezzo A, Menciassi A, Geng T, Saaj CM (2014) Crimped braided sleeves for soft, actuating arm in robotic abdominal surgery. Minim Inv Ther Allied Technol 24(4):204-210. doi:10.3109/13645706.2015.1012083

35. http://www.vascutek.com/

36. Chou C-P, Hannaford B (1996) Measurement and modeling of mckibben pneumatic artificial muscles. IEEE Trans Robot Autom 12(1):90-102

37. http://www.ndigital.com/medical/products/aurora/ 